\title{
Hormone replacement therapy increases levels of antibodies against heat shock protein 65 and certain species of oxidized low density lipoprotein
}

L. Uint ${ }^{1}$, O.C.E. Gebara ${ }^{2}$, L.B. Pinto ${ }^{2}$, M. Wajngarten ${ }^{2}$, P. Boschcov 3,4 , P.L. da Luz ${ }^{1}$ and M. Gidlund ${ }^{4}$
Correspondence

M. Gidlund

Laboratório de Imunofisiopatologia UP10, Departamento de Imunologia ICB-IV, USP

Av. Prof. Lineu Prestes, 1730

05508-9000 São Paulo, SP

Brasil

E-mail: gidlundm@usp.br

Research supported by FAPESP (Nos. 99/00158-4 and 01/13010-7), CNPq, LIM-38, and FINEP/FNDCT

(No. 66.95.0547.00)

Received February 5, 2002 Accepted January 29, 2003

\begin{abstract}
Unidades de ${ }^{1}$ Ateroscleroses, and ${ }^{2}$ Cardiologia Geriátrica, Instituto do Coração, Faculdade de Medicina, Universidade de São Paulo, São Paulo, SP, Brasil ${ }^{3}$ Departamento de Biofísica, Universidade Federal de São Paulo, São Paulo, SP, Brasil ${ }^{4}$ Departamento de Imunologia and Instituto de Medicina Tropical da Universidade de São Paulo, Instituto de Ciências Biomédicas IV, Universidade de São Paulo, São Paulo, SP, Brasil
\end{abstract}

\begin{abstract}
Hormone replacement therapy (HRT) reduces cardiovascular risks, although the initiation of therapy may be associated with transient adverse ischemic and thrombotic events. Antibodies against heat shock protein (Hsp) and oxidized low density lipoprotein (LDL) have been found in atherosclerotic lesions and plasma of patients with coronary artery disease and may play an important role in the pathogenesis of atherosclerosis. The aim of the present study was to assess the effects of HRT on the immune response by measuring plasma levels of antibodies against Hsp 65 and LDL with a low and high degree of copper-mediated oxidative modification of 20 postmenopausal women before and 90 days after receiving orally $0.625 \mathrm{mg}$ equine conjugate estrogen plus $2.5 \mathrm{mg}$ medroxyprogesterone acetate per day. HRT significantly increased antibodies against Hsp 65 (0.316 \pm 0.03 vs $0.558 \pm 0.11)$ and against $L D L$ with a low degree of oxidative modification $(0.100 \pm 0.01$ vs $0.217 \pm 0.02)(\mathrm{P}<0.05$ and $\mathrm{P}<0.001$, respectively, ANOVA). The hormone-mediated immune response may trigger an inflammatory response within the vessel wall and potentially increase plaque burden. Whether or not this immune response is temporary or sustained and deleterious requires further investigation.
\end{abstract}

Macrophages, activated T cells, B cells and immunoglobulins are found in atherosclerotic lesions, suggesting that autoimmunity plays a significant role in the pathogenesis of coronary artery disease (CAD) (1). Low density lipoprotein (LDL) and oxidative modifications of LDL particles contri-
Key words

- Hormone replacement therapy

- Postmenopause

- Oxidized LDL

- Heat shock protein

- Immune response

- Antibodies bute to the genesis and progression of atherosclerosis by promoting endothelial damage and amplifying the inflammatory response within the vessel wall. Highly oxidized LDL (oxLDL) is cytotoxic and may cause endothelial damage; however, less modified oxLDL is an immune stimulatory 
molecule and may up-regulate class II antigen molecules found on monocytes and therefore stimulate T cells (2). Oxidized LDL is also an antigen evoking antibodies against oxLDL and higher levels of antibodies against oxLDL have been found in patients with coronary atherosclerosis compared to normal controls (3).

Another antigen that may play an important role in atherogenesis belongs to the heat shock protein (Hsp) family. Hsp are highly conserved proteins synthesized when cells are exposed to stressful stimuli such as inflammation, infection and oxidizing agents.

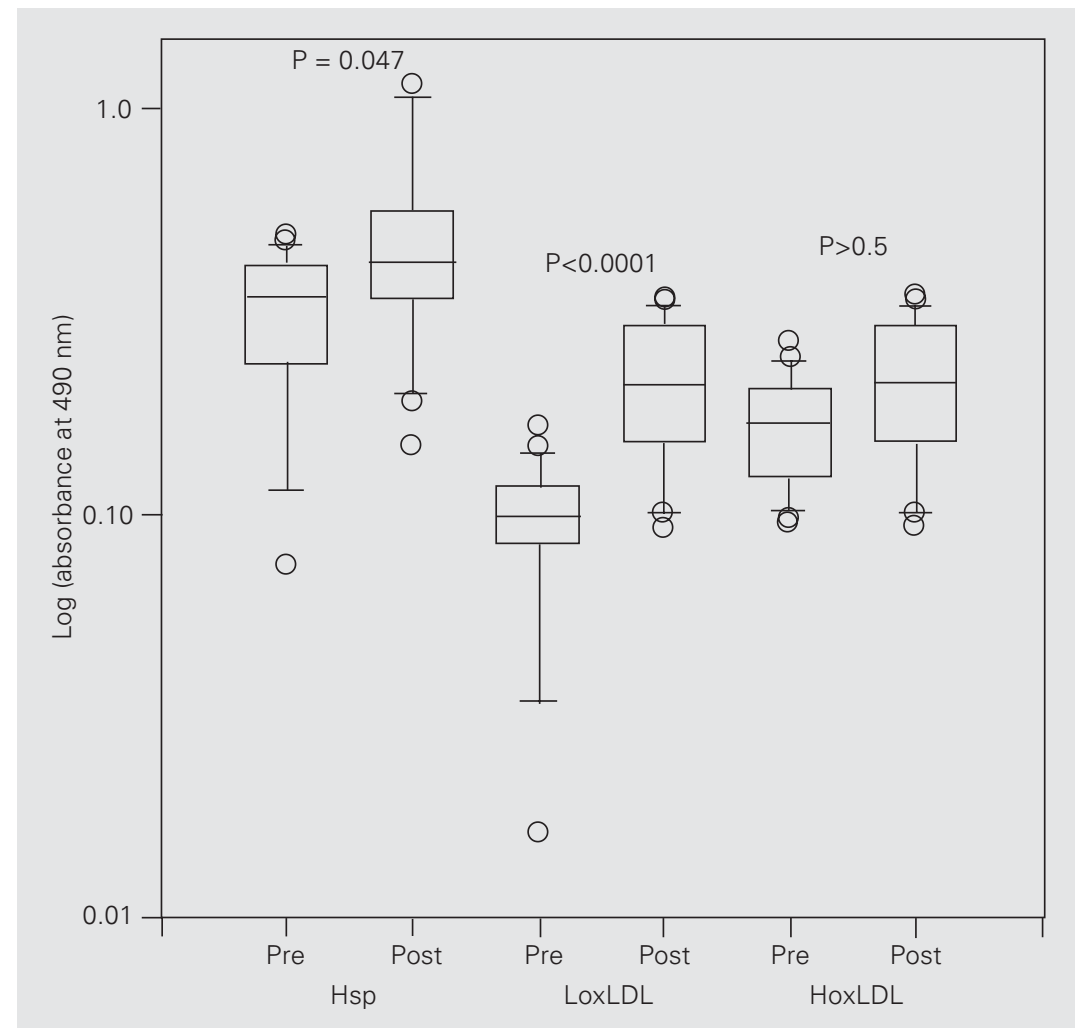

Figure 1. Reactivity of serum IgG antibodies against low density lipoprotein with a high (HoxLDL) and low (LoxLDL) degree of oxidative modification and heat shock protein (Hsp) before and after hormone replacement therapy. LDL was purified by ultracentrifugation and oxidized with $10 \mu \mathrm{M} \mathrm{CuSO}_{4}$ as described by Frostegard et al. (5). ELISA was performed with LoxLDL (TBARS: absorbance at $532 \mathrm{~nm}=0.051$ ), or HoxLDL (absorbance at $532 \mathrm{~nm}=0.71$ ) or Hsp (6). Plates were coated with $1 \mu \mathrm{g} / \mathrm{ml}$ antigen and blocked with $5 \%$ fat-free milk. Samples were diluted 1:500 in phosphate-buffered saline. Bound IgG was detected by adding a sorbent-purified rabbit peroxidase-labeled polyclonal anti-lgG antibody (1:3000, Dako A/S, Carpinteria, CA, USA). After incubation the reaction was developed by the addition of orthophenylenediamine (Sigma, St. Louis, MO, USA). The assay was read at absorbance of $490 \mathrm{~nm}$ in a microplate reader (BioRad, Hercules, CA, USA).
Increased expression of Hsp 60 has been reported on endothelial cells, macrophage and smooth muscle cells in human atherosclerotic plaques. Mean antibody titers of Hsp 60 were higher in CAD patients than in controls and were also related to disease severity (4).

It has also been shown that oxLDL induces Hsp in monocytes and therefore both antigens may be important for atherogenesis. However, little is known about how these antigens vary during the atherosclerotic process or how antiatherogenic therapy could alter their serum titers.

We studied 20 postmenopausal women (62 \pm 6.9 years) subjected to hormone replacement therapy (HRT) before and 90 days after receiving daily oral capsules of 0.625 $\mathrm{mg}$ equine conjugate estrogen plus $2.5 \mathrm{mg}$ medroxyprogesterone acetate. Serum samples were collected before and after HRT with written informed consent from patients recruited from the Geriatric Cardiology Unit, Heart Institute, University of São Paulo. The study was approved by the Ethics Committee of the Heart Institute. Patients had no previous record of CAD, all were nonsmokers and nondiabetic and none had received previous HRT. Serum samples were aliquoted and stored at $-70^{\circ} \mathrm{C}$ until analysis. Total cholesterol and high density lipoprotein (HDL) were determined by enzymatic and colorimetric methods such as cholesterol oxidase phenol aminoantipyrine and phosphatidic acid/magnesium chloride. Plasma triglyceride levels were determined with an enzymatic commercial kit (Abbott, South Passadena, CA, USA). LDL was estimated according to the Friedwald equation. All samples were tested at the same time and separately for antibodies against LDL with a low (LoxLDL) and high (HoxLDL) degree of oxidative modification and against a highly purified recombinant Hsp 65 by ELISA.

The results for the 20 participants in the study are shown in Figure 1 and Table 1. After 90 days, HRT promoted a significant 
reduction in total cholesterol levels $(230 \pm$ 40 vs $212 \pm 34 \mathrm{mg} / \mathrm{dl}, \mathrm{P}=0.004$, paired Student $t$-test) and LDL cholesterol (147 \pm 41 vs $123 \pm 33 \mathrm{mg} / \mathrm{dl}, \mathrm{P}=0.001$, paired Student $t$-test), and raised HDL cholesterol levels $(56 \pm 20$ vs $62 \pm 20 \mathrm{mg} / \mathrm{dl}, \mathrm{P}=0.004$, paired Student $t$-test); however, no significant change was found in plasma triglyceride levels $(135 \pm 63$ vs $136 \pm 55, \mathrm{P}=0.885$, paired Student $t$-test).

We found an increase in plasma levels of antibodies against Hsp $65(0.316 \pm 0.03 v s$ $0.558 \pm 0.11, \mathrm{P}=0.047, \mathrm{ANOVA})$ and against LoxLDL $(0.100 \pm 0.01$ vs $0.217 \pm 0.02$, $\mathrm{P}<0.0001$, ANOVA) after 90 days of HRT; however, no change was found in serum antibodies against HoxLDL $(0.171 \pm 0.05 v s$ $0.217 \pm 0.08)$. When reactive antibodies were determined pre- and post-therapy for each individual patient, no correlation was found between levels of antibodies against Hsp 65 and against anti-LoxLDL and anti-HoxLDL after HRT, strongly suggesting that they represent independent antibody reactivities (Table 1). However, a significant correlation was found between antibodies against Hsp 65 and HoxLDL at baseline before HRT $(\mathrm{P}=$ $0.02)$.

The exogenous administration of sex hormones to this relatively homogeneous group of women after a prolonged postmenopausal period provides a unique opportunity to analyze the systemic effects of hormonal stimuli. In contrast to the majority of reports on HRT using a prolonged study period, our aim was to access a more acute response to this treatment, i.e., 2-3 months. We studied lipoprotein profile changes and also antibody formation against two antigens implicated in atherosclerosis. HRT improved the lipid profile, reducing total cholesterol and LDL and increasing HDL, in agreement to previous reports (7).

The simultaneous measurement of antibodies against different antigens related to CAD yielded several interesting results. The analysis of two oxLDL preparations with different degrees of oxidative modification derived from the same LDL revealed a change in antibody reactivity against LoxLDL but not HoxLDL during the time of observation. The role of antibodies against anti-oxLDL in atherogenesis remains unclear. Antibodies against oxLDL have been correlated with the severity of CAD. However, in experimental atherosclerosis animal immunization with oxLDL antigens prevented foam cell formation. Our data showed the presence of at least two classes of antibodies against oxLDL and a significant increase of only one class after treatment. This finding supports previous data about the heterogeneity of the oxLDL antibody response and its dual beneficial and detrimental effects. No correlation was found between LDL and cholesterol levels. The entire LDL particle and/or cholesterol could harbor oxidative-induced antigenic determinants and constitute two potential sources of antigens. In a recent study, Heikkinen et al. (8) demonstrated that HRT did not alter serum levels of anti-oxLDL antibodies. However, in the cited study women were younger (mean age, 52 years) and were treated for a longer period of time. The antibodies were similar to HoxLDL used in the present investigation, in agreement

Table 1. Correlation between the presence of antibodies against low density lipoprotein with a high (HoxLDL) and low (LoxLDL) degree of oxidative modification and heat shock protein (Hsp) 65 before and after hormone replacement therapy (HRT).

\begin{tabular}{llll}
\hline \multirow{2}{*}{$\begin{array}{c}\text { Antibodies } \\
\text { measured against }\end{array}$} & \multicolumn{2}{c}{ Correlation } \\
\cline { 3 - 4 } & & Pre-HRT & Post-HRT \\
\hline LoxLDL & HoxLDL & 0.6 & 0.5 \\
LoxLDL & Hsp 65 & 0.4 & 0.2 \\
HoxLDL & Hsp 65 & 0.02 & 0.1 \\
\hline
\end{tabular}

Pre- and post-treatment (three months after HRT) samples were tested as described in Figure 1, and the correlation coefficent was determined using the Excel statistical program (Microsoft, San Diego, CA, USA). 
with our own findings since plasma levels of antibodies against HoxLDL were similar before and after HRT.

The same serum samples were tested simultaneously for Hsp antibodies. We found a significant increase in antibody response against Hsp 65 although the mechanism involved remains unclear. Nevertheless, this result agrees with a recent report demonstrating that estrogen was able to regulate the expression of Hsp (both the 90 and 70 subclasses) in human endometrium and several human cell lines (9). How antibodies against Hsp or cross-reactive antibodies against Hsp could influence the genesis and progression of CAD requires further investigation. However, the linkage between borderline hypertension, carotid intimal thickness and carotid atherosclerosis in response to higher serum titers of Hsp antibodies and the demonstration that these antibodies are cytotoxic to endothelial cells strongly suggest that Hsp may be involved in the genesis and progression of atherosclerosis. The correlation between HoxLDL and Hsp pretreatment could indicate the existence of cross-reactivity between antibodies. This potential antigenic cross-reactivity may lead to sizable apo B degradation and further antigenic stimuli.

We have shown that HRT can rapidly alter an ongoing humoral antibody response against potential cross-reactive or autoantigens. The link between oxLDL and Hsp may induce Hsp in monocytes and an inflammatory response within the vessel wall. In patients with known CAD, HRT may trigger the humoral response and increase antibodies against Hsp, and potentially aggravate an established lesion. Taken together, these findings suggest that initial HRT may be potentially deleterious. Whether or not the immune response is temporary or sustained and deleterious requires further investigation.

\section{Acknowledgments}

We thank Dr. Rolf Kiessling, Karolinska Institute, Stockholm, Sweden, who kindly provided Hsp.

\section{References}

1. Ross R (1999). Atherosclerosis - an inflammatory disease. New England Journal of Medicine, 340: 115-126.

2. Lusis AJ (2000). Atherosclerosis. Nature, 407: 233-241.

3. Holvoet P, Mertens A, Verhamme P, Bogaerts K, Beyens G, Verhaeghe R, Collen D, Muls E \& Van de Werf $F$ (2001). Circulating oxidized LDL is a useful marker for identifying patients with coronary artery disease. Arteriosclerosis, Thrombosis, and Vascular Biology, 21: 844-848

4. Zhu J, Quyyumi AA, Rott D, Csako G, Wu H, Halcox J \& Epstein SE (2001). Antibodies to human heat-shock protein 60 are associated with the presence and the severity of coronary artery disease. Evidence for an autoimmune component of atherogenesis. Circulation, 103: 1071-1075.

5. Frostegard J, Nilsson J, Haegerstrand A, Hamsten A, Wigzell H \& Gidlund M (1990). Oxidized low density lipoprotein induces differentiation and adhesion of human monocytes and the monocytic cell line U937. Proceedings of the National Academy of Sciences, USA, 87: 904-908.

6. Pockley AG, Wu R, Lemne C, Kiessling R, de Faire U \& Frostegard J (2000). Circulating heat shock protein 60 is associated with early cardiovascular disease. Hypertension, 36: 303-307.

7. Herrington DM, Reboussin DM, Branihan KB et al. (2000). Effects of estrogen replacement on the progression of coronary atherosclerosis. New England Journal of Medicine, 343: 522-529.

8. Heikkinen AM, Niskanen L, Yla-Herttuala S, Luoma J, Tuppurainen MT \& Komulainen M (1998). Postmenopausal hormone replacement therapy and autoantibodies against oxidized LDL. Maturitas, 29: 155-161.

9. Tang PZ, Gannon MJ, Andrew A \& Miller D (1995). Evidence for oestrogenic regulation of heat shock protein expression in human endometrium and steroid-responsive cell lines. European Journal of Endocrinology, 133: 598-605. 